\title{
La tolerancia del turismo y la transformación de una fiesta tradicional: actitudes de la población local
}

\section{The tolerance of tourism and the transformation of a traditional festival: attitudes of the local population}

\author{
Martha Marivel Mendoza Ontiveros (iD, Universidad Autónoma del Estado de México, \\ México.marivelmo@hotmail.com \\ Marcelino Alejo Pacheco, Universidad Autónoma del Estado de México, México. \\ marsalejo@yahoo.com \\ Roberto Rivera Pérez, Multiversidad Mundo Real Edgard Morin, A. C., México. \\ el roo17@hotmail.com
}

\section{RESUMEN}

La investigación de los impactos positivos o negativos de la actividad turística ha transitado por diferentes etapas, sin embargo, hasta el presente las investigaciones sólo se han limitado a mencionar los impactos y sus características cuantitativas sin lograr una comprensión profunda sobre su génesis y evolución. En el presente documento se expone cómo el uso de la etnografía, como método, puede posibilitar el entender y explicar las actitudes y comportamiento de la población residente de una comunidad del Estado de México con respecto a una celebración que ha logrado atraer la atención de un número significativo de jóvenes. El método ha permitido explicar cómo un fenómeno cuyo impacto podría calificarse como negativo para la población, se percibe, en los hechos, como positivo, ya que no altera o modifica los valores tradicionales de la población receptora.

Palabras clave: Turismo, impactos, fiesta tradicional, etnografía, Tepetlaoxtoc.

\section{ABSTRACT}

Research on the positive or negative impacts of the tourism activity has gone through different stages. However, until now, this research has been limited to mentioning the impacts and their quantitative characteristics without achieving a deep understanding of their genesis and evolution. This paper examines how the use of ethnography, as a method, may allow us to understand and explain the attitudes and behavior of the resident population of a community in the State of Mexico with respect to a celebration that has managed to attract the attention of a significant number of young people. The method enables us to explain how a phenomenon 
whose impact could be described as negative for the population, is actually perceived as positive, since it does not alter or modify the traditional values of the receiving population.

Keywords: Tourism, impacts, traditional celebration, ethnography Tepetlaoxtoc.

\section{INTRODUCCIÓN}

Comprender los impactos sociales y culturales del turismo en las sociedades receptoras es importante para los gobiernos a todos los niveles, ya que permite tomar medidas para reducir la probabilidad de una reacción negativa contra los turistas y el desarrollo turístico. Dado que los residentes de muchos destinos turísticos son una parte fundamental del producto turístico, las actitudes y comportamiento de los pobladores tienen una influencia considerable en el éxito o fracaso de un destino. La investigación sobre los impactos socioculturales del turismo en las comunidades es sustancial y continua, y aunque se han logrado avances en el área, no se han abordado algunos de los problemas más profundos que se enfrentan en los destinos turísticos, además son necesarias las actualizaciones periódicas y la revisión de las conclusiones propuestas con el fin de reducir las posibilidades de duplicación involuntaria.

Por otra parte, la mayoría de los trabajos publicados (Sharpley, 2014) ha sido realizada con metodologías cuantitativas, lo cual limita la capacidad de obtener una comprensión más profunda de los impactos; al quedar a nivel de "listas de impacto social" no permiten conocer cómo influyen en las actitudes tanto en la comunidad anfitriona como en los turistas, ni cómo modificarlas. En otros términos, si bien es importante saber qué impactos son motivo de preocupación para los residentes y un punto de partida para futuras investigaciones, la lista de impactos no proporciona una idea de por qué los residentes perciben tales impactos de manera particular y, por lo tanto, se pierde la oportunidad de cambiar percepciones. Lo que la lista de impactos no dice es por qué estos impactos son clasificados positiva o negativamente por los residentes por lo que se plantea que la investigación cualitativa en este campo puede ofrecer una comprensión más profunda y holística (Deery, Jago y Fredline, 2012).

El artículo que se presenta a continuación expone los resultados de una investigación en la que se utilizó un enfoque cualitativo a través del registro etnográfico de una fiesta tradicional en un poblado mexicano. A esta fiesta, en los últimos años, empezaron a concurrir cientos de jóvenes, motivados por la bebida tradicional -pulque-, que en ella se "regalaba". Se describen los problemas surgidos a partir de su llegada, así como los impactos percibidos por los pobladores locales y sus actitudes hacia los visitantes. El uso del método cualitativo posibilitó examinar con mayor profundidad las actitudes de los locales y vincularlas con los valores y las características intrínsecas de la cultura de los residentes en general, y de su modelo de ser hombre y mujer para proponer una explicación alternativa. La etnografía como método pretende abordar el fenómeno de estudio desde la perspectiva del individuo investigado, por lo que la descripción densa (Geertz, 1987) y la presentación de testimonios recabados permiten elaborar los conceptos y definiciones durante el proceso mismo.

Este documento presenta en la primera parte la revisión teórico-conceptual y metodológica con las que se han abordado los impactos del turismo, su percepción y las actitudes de 
las poblaciones receptoras para subrayar sus avances y limitaciones, al tiempo que se justifica el planteamiento teórico metodológico utilizado en esta investigación. A partir de lo anterior, se realiza la descripción etnográfica de la festividad denominada por los turistas "Feria del Pulque" y las percepciones sobre los cambios y vicisitudes experimentadas por la llegada de los jóvenes que buscan embriagarse con pulque. Finalmente se propone una explicación a la paradójica actitud positiva de los lugareños, a pesar de los inconvenientes que produce la llegada de los turistas, explicación fundamentada en los valores compartidos y modelos de masculinidad y femineidad.

\section{PERSPECTIVA TEÓRICA-CONCEPTUAL}

La planificación y gestión de un destino turístico es una cuestión muy compleja (Mathieson y Wall, 1982) debido al número de instancias implicadas en el proceso. Tales instancias, entre ellas la población residente (Gunn, 1988), se ven afectadas por el mismo desarrollo turístico.

De manera particular, el respaldo o la buena voluntad de los residentes es esencial para el correcto desarrollo turístico por diversas razones (Gursoy, Jurowski y Uysal, 2002). Cada día la experiencia demuestra que una población local abierta y hospitalaria es un factor intangible en el éxito de todo proyecto turístico, ya sea porque es prestadora de servicios (alimentos, hospedaje, transporte u otros) o porque es parte de la oferta turística (eventos culturales, atracciones y demostración de tradiciones). La comprensión de la percepción local y los factores que influyen sobre las actitudes tanto de los lugareños como de los visitantes, aunado a los servicios de seguridad y elementos de comodidad, son fundamentales para obtener apoyo para el desarrollo turístico.

Cuando los niveles de irritación o frustración de la sociedad receptora aumentan pueden darse manifestaciones de rechazo de manera explícita, como ocurrió en el municipio de Lloret de Mar municipio en España. En este caso los habitantes salieron a la calle para reclamar un cambio de modelo turístico y denunciar que están hartos de la mala imagen que se da de la población al resto de Europa (Escriche, 2011). Otro caso fue el fenómeno de turismofobia reportado en Barcelona en 2017 (Sierra, 2017). Por otro lado, el descontento puede ser latente, un ejemplo de ello es el destino mexicano de Cancún y el caso particular de los Spring Break (Monterrubio y Andriotis, 2014).

Mathieson y Wall (1982) señalan que muchos estudios hacen referencia a la presencia de impactos sociales positivos y negativos, sin embargo la mayoría no ha aportado información sobre la naturaleza de tales impactos. Por tal motivo propusieron que la investigación se dirigiera más explicitamente a la determinación de las percepciones y actitudes de la población residente sobre la presencia y el comportamiento de los turistas, a partir de ello han aparecido numerosos estudios centrados en las actitudes de los residentes hacia los turistas y el desarroIlo del turismo (Ap y Crompton 1993; Hernández, Cohen y García, 1996; Johnson, Snepenger y Akis 1994; Lankford 1994; Lankford y Howard 1994; Akis, Peristianis y Warner, 1996; Lankford, Williams y Knowles-Lankford 1997; Besculides, Lee y McCormick, 2002; Choi y Murray, 2010; Choi y Sirakaya, 2005; Andereck, Valentine, Knopf y Vogt, 2005; Faulkner y Tideswell, 1997; Fredline y Faulkner, 2000; Gursoy y Kendall, 2006; Gursoy y Rutherford, 2004; Gursoy, Jurowski 
y Uysal, 2002; Haralambopoulos y Pizam, 1996; Hunt y Stronza, 2014; Jurowski y Gursoy, 2004; Vargas, Porras y Plaza, 2011; Wang y Pfister, 2008; Weaver y Lawton, 2001, 2013; Woosnam, 2012; Yu, Chancellor y Cole, 2011; Zamani-Farahani y Musa, 2012).

De acuerdo con Deery et al. (2012), la investigación sobre los impactos sociales del turismo ha pasado por varias etapas de desarrollo. La primera de ellas corresponde a las definiciones y desarrollo de conceptos (por ejemplo, Milman y Pizam 1988; Lui, Sheldon y Var, 1987), ya que al igual que en todas las áreas de investigación emergentes, inicialmente hay necesidad de definiciones para establecer los límites del objeto de estudio. Esta exigencia hizo alejar la atención del enfoque económico que dominó gran parte de los primeros estudios sobre los impactos del turismo. Sin embargo, los debates sobre las definiciones de palabras clave tales como residentes, impactos sociales, comunidad, percepciones y actitudes continúan. Gran parte de la discusión en la literatura gira en torno al término residentes, en tanto otros se centran en el significado de actitudes. En varios estudios actitudes y percepciones suelen utilizarse como sinónimos (Andereck, Valentine, Knopf y Vogt, 2005; Dyer, Gursoy, Sharma y Cater, 2007; Kayat, 2002; Ross, 1992; Sharma, Dyer, Carter y Gursoy, 2008; Small, 2007) y se argumenta que describen la experiencia de una persona en el mundo. Para otros investigadores, las actitudes se entienden como reacciones (Fredline y Faulkner, 2000) y también se ha utilizado como opiniones (Williams y Lawson, 2001).

La segunda etapa corresponde al desarrollo de modelos (Doxey, 1975; Butler, 1980; Matheison y Wall, 1982; Perdue, Long y Allen, 1990) en un intento de mostrar las relaciones entre las percepciones de los residentes sobre los impactos sociales del turismo y las variables que intervienen en ellas tales como mejores servicios y oportunidades de recreación (Mathieson y Wall, 1982), el crimen, la congestión y el trastocamiento de la vida social (Ap, 1992), apoyada por la teoría del intercambio social (Blau, 1964; Emerson, 1976). La teoría del ciclo de vida de un destino (Butler, 1980) también se ha utilizado para sustentar la investigación sobre el impacto social, aunque esta teoría se ha encontrado menos útil, ya que asume la homogeneidad dentro de las comunidades y el supuesto de un recorrido unilineal por una serie de etapas. Con el tiempo, los modelos desarrollados durante esta segunda etapa se ampliaron para proporcionar una base para pruebas estadísticas. Los trabajos de Ap y Crompton $(1993,1998)$ y de Andereck y Vogt (2000) marcaron el comienzo de la tercera fase, que se ha caracterizado por el diseño y desarrollo de instrumentos de medición. Esta fase continuó para probar y perfeccionar los instrumentos utilizados para cuantificar las actitudes en la cuarta fase (Fredline y Faulkner, 2000; Choi y Sirakaya, 2005).

Asimismo, Deery et al. (2012) sostienen que, aunque la investigación sobre las percepciones y actitudes sobre los impactos del turismo es abundante, todavía hay debates sobre las definiciones (por ejemplo, Yen y Kerstetter, 2009), las variables que influyen en ellas (Jurowski y Gursoy, 2004; Smith y Krannich, 1998) y los métodos utilizados (Gursoy, Jurowski y Uysal, 2002; Huh y Vogt, 2008). En otras palabras, la investigación sobre los impactos sociales del turismo parece estar en un estado de desarrollo detenido. Si bien existe un acuerdo razonable en cuanto a la naturaleza de los impactos (sobrepoblación, comportamiento perturbador del turista, mayores tasas de empleo, entre otros) y las variables que influyen en la percepción de los residentes, la lista de impactos no proporciona una idea de por qué los residentes perciben de una manera particular y, por lo tanto, se pierde la oportunidad de cambiar dicha 
percepción. La lista de impactos no da más información sobre por qué estos impactos son clasificados positiva o negativamente por los residentes, asimismo la investigación cuantitativa que ha predominado limita una comprensión profunda de las causas de tales percepciones y las consecuencias subsecuentes (Northcote y Macbeth, 2005; Sharpley, 2014).

Muchas investigaciones realizadas han utilizado la teoría del intercambio social y, aunque esta teoría ha sido una herramienta útil para explorar los impactos sociales, ha llevado al enfoque de "lista" que predomina en la literatura, que implica un conocimiento superficial. Por lo anterior, Deery et al. (2012) plantea el uso de otras bases teóricas desarrolladas por la antropología y los paradigmas cualitativos, por ejemplo, la etnografía pata tener un acercamiento desde la perspectiva de las personas involucradas.

Una limitación adicional de la teoría del intercambio social es que está estrechamente vinculada a la teoría de la elección racional (Fishbein y Ajzen, 1975) debido a que concibe al individuo como un homo economicus, hombre racional que cuenta con información completa, persigue su propio interés, quiere maximizar beneficios y reducir costos, es decir, un sujeto será racional cuando es capaz de ponderar la relación entre los costos y los beneficios que espera recibir de la acción que emprende, lo cual significa que no realizará ninguna acción si cree que no recibe beneficio alguno. No obstante, Weber (1964) afirma que la plena conciencia de las acciones de un individuo es un caso límite y que, en la inmensa mayoría de las situaciones, es parcialmente consciente de lo que hace. En un número considerable de casos, la acción real sucede con oscura seminconsciencia o plena inconsciencia de su sentido nombrado. El agente más bien siente de un modo indeterminado que sabe o tiene clara la idea y actúa por instinto o costumbre. Sólo ocasionalmente algunos individuos tienen conciencia del sentido (sea racional o irracional) de la acción.

Así, Weber (1964) asevera que la acción social, como cualquier otra acción, puede dividirse en cuatro categorías en función de la orientación del proceder social: 1) la acción racional con arreglo a fines o instrumental; 2) la acción racional con arreglo a valores; 3) la acción afectiva que es determinada por emociones y estados de ánimo; y 4) la acción tradicional que es determinada por una costumbre arraigada. Para Weber la acción humana es un objeto que, en tanto que muestra nexos y regularidades que son causales, puede explicarse, por vía de la comprensión, de un modo intersubjetivo y, por lo tanto, plenamente objetivo, esto es, a través de un medio metodológico que cumple con las condiciones para la validación objetiva del conocimiento producido. Bajo este precepto para dar cuenta del sentido de la acción social es necesario atender a sus causas sociales y no a los motivos psicológicos internos de los individuos como se ha venido realizando en los estudios sobre la percepción de los impactos del turismo y las actitudes asociadas a ellos. Así, la comprensión de la acción es una forma de interpretación del sentido que orienta la conducta externa de los actores y hacia las regularidades o leyes.

A partir de lo anterior, en este documento se describirán los resultados de una investigación en la que se estudiaron las percepciones y las actitudes de los habitantes de un pueblo mexicano, Tepetlaoxtoc, hacia los turistas que acuden a una de sus fiestas tradicionales más significativas, Día del Tlachiquero, a partir de un estudio cualitativo. Como un paradigma, la investigación cualitativa corresponde a un conjunto de supuestos ontológico, epistemológico y metodológico. Se empleó la etnografía, como método, con el propósito de comprender los 
problemas desde la perspectiva del actor, se obtuvieron datos de la observación participante y testimonios de los pobladores en varias visitas a la población. Dichas estancias iniciaron en el segundo semestre de 2008 y la observación de la festividad en el 2009, 2010, 2011 y 2016. Gracias al método etnográfico se ofrece una descripción detallada de la fiesta patronal, de las personas que participan en ella y de su comportamiento.

El enfoque etnográfico se apoya en la convicción de que las tradiciones, roles, valores y normas del ambiente en que se vive se van interiorizando poco a poco y generan regularidades que pueden explicar la conducta individual y de grupo de forma adecuada. Los miembros de un grupo étnico, cultural o situacional comparten una estructura lógica o de razonamiento que, por lo general, no es explícita, pero que se manifiesta en diferentes aspectos. Es un proceso dirigido hacia el descubrimiento de muchas historias y relatos idiosincrásicos, pero importantes, contados por personas reales, sobre eventos reales, en forma real y natural. Este enfoque trata de presentar episodios que son porciones de vida documentados con un lenguaje natural y que representan lo más fielmente posible cómo siente la gente, qué sabe, cómo lo conoce y cuáles son sus creencias, percepciones y modos de ver y entender (Guber, 2004). Los fenómenos son estudiados in situ y las teorías emergen de los datos empíricos relacionados con las estructuras de los eventos y con la vida de las personas, así como es vivida en su autenticidad y espontaneidad.

Igualmente, no existe hipótesis o problema inicial de forma explícita (Velasco y Díaz de Rada, 1997). La investigación etnográfica, en el sentido estricto, ha consistido en la producción de estudios analítico-descriptivos de las costumbres, creencias, prácticas sociales y religiosas.

El trabajo de campo etnográfico asume que el ser humano es el mejor instrumento para estudiar los grupos humanos, o, expresado menos retóricamente: la mejor estrategia para el análisis de los grupos humanos es establecer y operar relaciones sociales con las personas que los integran por medio de la observación y entrevista como dos modos básicos de obtener y producir información, es decir, observar y preguntar. Es clásico justificar la complementariedad de ambas técnicas con el argumento del control, de la fiabilidad, o de la significatividad de los datos. La observación, suele decirse, proporciona el contraste de la realidad -de la objetividad-a lo que a veces imaginativamente se comunica en la entrevista. La entrevista, a su vez, proporciona sentido a las acciones a veces incomprensibles que se observan, o corrige las inferencias a veces precipitadas que se obtienen por observación (Velasco y Díaz de Rada, 1997).

\section{PERSPECTIVA ETNOGRÁFICA}

El municipio mexicano de Tepetlaoxtoc de Hidalgo se localiza en el extremo oriental del Valle de México y del Estado de México. La distancia aproximada a la capital del país, es de 100 kilómetros, cuenta con una población 27,944 habitantes (CONEVAL, 2015).

Su cabecera municipal, Tepetlaoxtoc de Magdalena como muchos pueblos de México, conserva aún una serie de fiestas patronales organizadas por las tradicionales mayordomías, fiestas que caracteriza a la población católica. La más grande e importante en todo el municipio de Tepetlaoxtoc es la fiesta del 20 de enero en honor de San Sebastián Mártir, en la cual se presentan nueve asociaciones de mayordomos. La celebración comienza el domingo previo 
al 20 de enero con la participación de la mayordomía de los mexicanos y la del 19 de enero. En adelante la festividad se reparte entre las mayordomías de siete gremios o cofradías, que habrán de conformarse según la tradición popular, comenzando con los cirios, las molenderas, los gañanes, los tlachiqueros, los albañiles, los comerciantes y los arrieros. Así las agrupaciones se encargarán de aportar lo necesario para la realización de los festejos para agradecer o pedir los favores del santo patrón.

Varias de las ocupaciones han desaparecido con el paso del tiempo, también han decaído gran parte de estos gremios y con ello se han transformado las tradiciones originales. No obstante, las cofradías de los gañanes, tlachiqueros, albañiles y arrieros realizan, el día que les corresponde, un simulacro o performance que recrea las condiciones laborales que enfrentaban.

En la explanada principal del templo de San Sebastián Mártir se coloca una muestra gastronómica y artesanal que los visitantes pueden disfrutar y en lo que podría fungir como el atrio de San Sebastián, frente a la capilla, se organizan espectáculos pirotécnicos y bailes. Además, cada año, es convocado un elenco artístico para amenizar la fiesta grande.

Para la presente investigación nos concentraremos en la mayordomía del gremio de los tlachiqueros por ser el día que arriban más personas a Tepetlaoxtoc. Durante los días restantes la presencia de forasteros es reducida. Los tlachiqueros son personas que se han dedicado a recolectar la sabia de maguey para que, una vez fermentada, se convierta en pulque ${ }^{1}$. La primera referencia que se tiene de ellos proviene de la novela histórica Los Bandidos de Río Frío de Manuel Payno (Rivera Pérez, 2013). La participación de los tlachiqueros comenzó aproximadamente en 1930, cuando en el Estado de México se prohibió esta labor. Como resultado la actividad se volvió ilegal, propia de contrabandistas y traficantes. En el simulacro de los tlachiqueros se colocan 16 magueyes repartidos por la calle principal y se representa extraer aguamiel o el jugo del maguey para luego entregar gratuitamente pulque a todo aquel que lo acepte.

El simulacro de los tlachiqueros se realizó sin novedad hasta 2005 o 2006, año en que un joven publicó en su blog sus vivencias en la festividad bautizándola como la Feria del Pulque en Tepetlaoxtoc. A partir de ese momento empezaron a subirse a Youtube vides, fotografías y bailes con el mismo título (Rivera Pérez, 2013). Como respuesta, pronto varias personas empezaron a escribirle para preguntar cómo se llegaba a este pueblo del Estado de México. en los años siguientes empezaron a arribar jóvenes con la idea de que iSe regalaba pulque!

Los operadores de autobuses particulares vieron en la festividad la oportunidad de un gran negocio, como resultado propusieron viajes redondos especiales a Tepetlaoxtoc con motivo de la denominada Feria del Pulque, de este modo empezaron a llegar visitantes de estados aledaños como: Veracruz, Querétaro, Hidalgo y Puebla; así como de Toluca, la capital del estado. A partir de ese momento Tepetlaoxtoc se hizo famoso y se ha calculado la llegada de hasta 5,000 personas ese día (Rivera Pérez, 2013).

1. Bebida alcohólica de alta graduación, espesa y de color blanco, que se obtiene de la fermentación del jugo del maguey. 
En 2007 el número de visitantes con ganas de embriagarse aumentó considerablemente. Los problemas resultantes de tal aglomeración hicieron que se pensara en cambiar de día del festejo, sin embargo, el cambio no se llevó a cabo por el argumento de que se rompería la tradición. Para el año siguiente se intentó frenar el exceso de visitantes con la estrategia de vender el pulque tras haber regalado una parte proporcional (Rivera Pérez, 2013).

En los años subsecuentes también aumentó el número de personas detenidas por faltas administrativas, por lo que en 2011 se implementó un operativo policial en los accesos al centro de Tepetlaoxtoc. El objetivo era tratar de detener el arribo de los narcomenudistas que comercializarían en los espacios públicos de la cabecera municipal. También arrestaron a toda persona ebria y a aquellos que portaban armas punzocortantes y/o de fuego (Rivera Pérez, 2013).

El día de la fiesta, aproximadamente a las nueve de la mañana, comienzan a llegar jóvenes cuyas edades oscilan entre 15 y 21 años, quienes inmediatamente acuden a las cantinas improvisadas que venden cerveza. Tiempo después llegan algunos tlachiqueros y empiezan a vender litros de pulque. Paulatinamente las calles del centro de Tepetlaoxtoc se convierten en un centro juvenil.

Para el mediodía, los visitantes presionan a los organizadores para que inicie el simulacro, pero solamente se realiza en algunos de los 16 lugares elegidos para el efecto, a razón de la excesiva demanda de pulque. Para la hora de la comida se observan ya jóvenes ebrios y dormidos en los jardines. Aproximadamente a las cinco de la tarde, comienzan a repartirse litros y litros de la bebida embriagante. Sin mencionar, que la venta y consumo de bebidas alcohólicas comenzó antes de las 9:00 am.

Al caer la noche da comienzo la música y los jóvenes suben a una plataforma para bailar exaltando el contacto físico con su pareja (heterosexual u homosexual), algunas jóvenes se tocan los senos y se levantan las blusas o bajan parcialmente los pantalones para mostrar su ropa interior (Rivera Pérez, 2013). Al anochecer, aprovechando la oscuridad, algunas parejas sostienen relaciones sexuales o consumen drogas en las inmediaciones.

Es común que durante la fiesta sean detenidos quienes protagonizan pleitos y riñas, hacen necesidades fisiológicas en la vía pública y cometen faltas a la moral (striptease, actos sexuales en pareja o en grupo), todo ello debido a la desinhibición causada por el alcohol y el valor social que otorga el anonimato en esta región. Por lo anterior, algunos elementos del cuerpo de policía municipal de Tepetlaoxtoc, a partir del 2010, comenzaron a llamar a esta festividad El Pulquetón.

La situación descrita ha provocado, por un lado, que las personas que habitan en las calles aledañas a la capilla de San Sebastián Mártir no participen como antes de la llegada de los jóvenes en la fiesta y prefieran quedarse en casa y, por otro, que muchos de los residentes se molesten por el desorden y el cambio del sentido de la festividad. Cabe mencionar que en las inmediaciones de la capilla se encuentra una escuela de educación básica que ese día suspende las clases ya que los residentes no permiten que los niños salgan a la calle. 


\section{IMPACTOS PERCIBIDOS POR LOS RESIDENTES}

El caso aquí descrito generó algunas reflexiones sobre las cuales avanzar. En primer lugar, es evidente que la fiesta patronal de Tepetlaoxtoc posee un nivel de singularidad tal que se convirtió, sin proponérselo, en un atractivo turístico. A diferencia de muchos pueblos que buscan atraer turistas para lo cual se valen de diversas estrategias, en este caso, llegaron sin esperarlos. No obstante, ante tal suceso no hubo anticipación ni gestión para evitar los problemas ocasionados por los visitantes, o bien para aprovechar su llegada.

Según los hallazgos de otros estudios sobre los impactos generados por el turismo, el económico es el principal beneficio percibido por la población local. Sin embargo, la apreciación de los testimonios de las personas residentes en la cabecera municipal es que no hay tal ganancia ni en lo individual ni en lo colectivo. Los comercios que se instalan en la vía pública los días que dura la festividad pertenecen a foráneos, quienes tienen la capacidad económica para pagar el derecho o permiso que les autoriza colocar su negocio temporalmente. Por el contrario, los comerciantes locales suelen no vender sus productos esos días.

Además, en los testimonios se sostiene que el Día del Tlachiquero hay un incremento en el precio de muchos productos y servicios. Por ejemplo, el pasaje desde la Ciudad de México aumenta hasta $100 \%$.

En orden de importancia, otro tipo de efectos percibidos, según la literatura, son los ocasionados sobre el medio ambiente. Cualquier persona puede observar la proliferación de basura en todo el espacio público usado como escenario para la fiesta. Al día siguiente amanece un exceso de residuos sólidos, sobre todo vasos desechables y se observa además vómito y orines por doquier. Los jóvenes que arriban a la fiesta se sientan, acuestan o transitan en las áreas verdes causándoles daño. Esta situación la advierten los habitantes de Tepetlaoxtoc en sus testimonios.

Asimismo, el incremento de tráfico vehicular se percibe por los residentes como una oportunidad para hacer negocio, pues la experiencia les dice que los automóviles que se quedan en la vía pública ese día pueden sufrir daños o robos parciales, por lo que es más seguro dejarlos en un estacionamiento vigilado a cambio de un pago. Sin embargo, en esos lugares se permiten los encuentros sexuales que el momento, la situación de anonimato y la embriaguez facilitan.

Otra consecuencia de la llegada de visitantes el Día del Tlachiquero es el temor entre los residentes porque perciben un aumento de la inseguridad. Los habitantes locales han dejado de asistir a la festividad y prefieren quedarse en casa ante los peligros que ocasionan las constantes riñas. Por medio de la observación se constató que los residentes que asisten, en términos generales, son hombres jóvenes o solteros que van en busca de una aventura sexual ocasional. Las mujeres no asisten y menos las jóvenes solteras. Pocas mujeres de la localidad acuden a condición de ir acompañadas de un hombre: padre, hermano o marido.

A partir de los testimonios recopilados, podría argumentarse que los visitantes generan más costos que beneficios a la sociedad local, por lo que en años recientes se han llevado a cabo consultas ciudadanas para decidir la instauración de la ley seca el Día del Tlachiquero. Dicho ejercicio no deja de estar sesgado ya que los anuncios impresos que invitan a manifestarse 
Ilevan la leyenda: "Por el rescate de nuestras tradiciones", lo cual retoma la postura de quienes no están de acuerdo con los excesos derivados por el abuso del alcohol. Como resultado, cada vez que se ha realizado una consulta, la mayoría de los votos pide que no se permita la venta de bebidas alcohólicas durante la festividad, ante la molestia de las minorías porque el atractivo de ingerir bebidas alcohólicas atrae a los jóvenes.

No obstante, a pesar de los problemas que los residentes atribuyen a la llegada de visitantes a su celebración, en la mayor parte de los testimonios declaran que no desean modificarla para hacerla un atractivo turístico. Algunos lugareños propusieron que se llevara a cabo en un lugar exprofeso para recibir a los turistas, fuera de la localidad, para que ellos continuaran su fiesta como siempre, dicha propuesta no prosperó. Otros plantearon mover de fecha la celebración, pero se argumentó que ello afectaría al orden cósmico y fue desechada. No obstante, los habitantes de Tepetlaoxtoc muestran molestia cuando escuchan Ilamar a su festividad Feria del Pulque, manifiestan que les genera una mala imagen y que sus tradiciones han sido modificadas por los visitantes.

Durante el trabajo de campo se escuchó a los habitantes de Tepetlaoxtoc considerar que debe seguir realizándose su fiesta tradicional. Manifestaron una actitud favorable hacia los visitantes, paradójicamente, les agrada la visita de estos jóvenes, esto es, quieren que sigan acudiendo personas de otros lugares a su pueblo. Tales resultados evidencian la debilidad de la teoría del intercambio social, pues en consonancia, después del balance negativo entre costos y beneficios era de esperar que la actitud hacia la visita de dichos jóvenes con motivo del Dia del Tlachiquero fuera también negativa, sin embargo, no resultó así, no hay una oposición contundente a que su pueblo sea visitado por otras personas durante este tiempo.

Entonces, ¿qué alternativas existen para tratar de interpretar estos resultados? Una vía, tomando la propuesta de Deery et al. (2012), fue avanzar por el aspecto social y no por el planteamiento psicológico como se ha llevado a cabo con las percepciones y actitudes de la sociedad residente. De este modo, se interpreta a partir del modelo de ser hombre y mujer asociado a una moral sexual, es decir, a los valores socioculturales imperantes en Tepetlaoxtoc.

El principio social imperante en este pueblo mexicano impone que sean los hombres quienes preferentemente deberán ser ubicados en los espacios para la sociabilidad, la cual se torna totalmente masculina como las cantinas, tiendas de abarrotes que permitan el consumo de bebidas alcohólicas, los bares, la esquina de la calle en compañía de otros varones, el puesto de tacos, el billar del pueblo, las canchas de futbol y frontón en los espacios en donde se desarrollan las prácticas y los juegos de la masculinidad. Los principios que participan en la construcción de la identidad masculina son los jaripeos, rodeo, palenques, carreras de caballos, concurso de caballos bailadores y las charreadas. Cada uno de estos eventos sirve para lucir el nombre de la familia a la que se pertenece, es decir, apelar al principio de persona sobre individuos en un espacio casi completamente masculino; asimismo, permite exhibir los capitales simbólicos (mujeres acompañantes y animales para la justa), materiales (prendas, carros y otras propiedades), capitales sociales (¿A quién conoces?, pero principalmente ¿Quién te reconoce?), políticos y económicos (las apuestas).

"Un hombre tepetlaoxtoqueño siempre debe saber lo que tiene qué hacer y tener la noción de cómo hacerlo" (Juan, 28 años). 
En ese sentido, toda la gama de capitales y medallones se presenta y exhibe frente a las mujeres no oriundas de la región, las cuales al verse seducidas por el contexto acceden más rápidamente a la intimidad sexual. Intimidad que se convierte en un medallón más de la masculinidad.

"En los jaripeos hay mucho ligue ahí, después de que haces tú monta" van las chavas y se te acercan. Con el puro hecho de que te vean ahí en el ruedo, ellas se fijan y te hacen la plática. Entonces pues tú les contestas y la ocasión se presta para bailar, después sí se presta la ocasión (para tener un acto sexual), pues les preguntas: ¿Sí quiere ir a un lugar más apartado?, iya sabes, el hombre llega hasta donde la mujer lo permite! ¿No? Lo mismo ocurre en los gallos, con el hecho de estar en el palenque entrando y saliendo de la arena, ellas se te acercan. También cuando te ven (las mujeres) botudito ${ }^{3}$, arregladito con sombrero y tu ropita vaquera, las muchachas se te acercan, les gusta. También si te ven alegre, que bailas y que te diviertes" (Roberto, 25 años).

Los varones residentes solteros, divorciados o separados llegan a la celebración con su propia botella de licor, pues ir a una fiesta en donde regalan pulque es "estar muy fregado" $\mathrm{y}$ constituye un deshonor. En Tepetlaoxtoc, los hombres son asiduos a las bebidas embriagantes. En una reunión de amigos, una botella suele terminarse en 20 minutos, se considera que platicar es "perder el tiempo" y quien recibe el vaso de una botella debe quedarse hasta que se acabe. Por lo anteriormente descrito puede comprenderse que resulte normal en Tepetlaoxtoc el uso y abuso de bebidas embriagantes durante la fiesta y que ello constituya un atractivo para muchos jóvenes. La norma no se altera o modifica durante las festividades.

Las mujeres solteras de esta comunidad no participan de la fiesta o participan en actividades en las que siempre se encuentre bajo el escrutinio de los padres, hermanos y demás familiares varones que estén relacionados por filiación o parentesco ritual con ella. La mujer joven en este poblado mantiene relaciones de noviazgo a escondidas de su familia y se le exige llegar virgen al matrimonio, por ello los hombres deben vigilarla para que se mantenga intacto el honor de su familia como lo etnografió Pitt-Rivers en Andalucía, España (1979). Los varones, por el contrario, pueden y deben salir en búsqueda de aventuras sexuales de las que aladean después. Las mujeres que acuden a la fiesta en su calidad de visitantes anónimas van en busca del vaquero sexualmente atractivo. Nuevamente la norma imperante en la comunidad no se ve alterada.

Es así que, es posible interpretar que en los días de la festividad la vida cotidiana de los residentes locales se ve perturbada y que existe afectación a sus buenas costumbres y moral como una consecuencia de lo ocurrido durante la festividad, de acuerdo con los testimonios recabados. Sin embargo, en los hechos, la visita de jóvenes que buscan embriagarse con pulque y mantener relaciones sexuales ocasionales se corresponde con los valores de la cultura tepetlaoxtoquense y representa una oportunidad para los hombres de la localidad para probar su hombría, estereotipo que constantemente se le exige a casi todo hombre en la cultura patriarcal mexicana, pero que a su vez está constantemente impugnando y renegociando al interior

2. Acto de montar caballo o toros.

3. Calzar botas vaqueras. 
de las relaciones de género que se realizan dentro del municipio de Tepetlaoxtoc. Si bien hay afectación a una festividad tradicional, las acciones sociales llevadas a cabo corresponden a la acción tradicional que fortalece y confirma los valores compartidos con respecto a la forma de ser hombre y mujer. De otra forma la hombría no podría ser probada, así como tampoco el recato y pudor para las mujeres residentes. Los otros, los visitantes, hombres o mujeres pueden permitirse los excesos de alcohol o sexo (heterosexual, homosexual, bisexual extramatrimonial), esto afianza los valores de toda una sociedad, la festividad permite exhibir la estructura social en términos de Turner (2007). El comportamiento de los visitantes varones corresponde al modelo de masculinidad de esta comunidad mexicana, a quienes se les prescribe; mientras que el comportamiento de las mujeres visitantes corresponde a lo que se les prohíbe a las lugareñas.

\section{CONSIDERACIONES FINALES}

Los datos obtenidos en este estudio nos permiten revisar la tendencia presente en el ámbito académico de considerar al turista como el villano de la historia, como depredador y provocador de desorden. Un análisis superficial de este caso conduciría a concluir que esto sucede en Tepetlaoxtoc en el Día del Tlachiquero, no obstante, la relación visitante-visitado debe pensarse dentro de las estructuras y procesos socioculturales más amplios, en una relación dialógica; oportunidad para llevar a cabo no solo intercambios económicos, sino sociales y sexuales. De alguna manera estos intercambios y negociaciones permiten afianzar los valores de la moral sexual prevaleciente entre los visitados, por lo que los visitantes se convierten en un elemento esencial para recrearlos y reforzarlos, independientemente de que un análisis de costo-beneficio los defina como depredadores.

Por lo anterior, la percepción de los impactos del turismo, la relación visitante-visitado y las actitudes de las sociedades receptoras hacia ellos debe ser analizada en función de una serie de circunstancias, favorables y desfavorables. El caso aquí analizado muestra que deben estudiarse las dinámicas promovidas por la llegada de turistas, no de forma discreta y aislada, sino dentro del conjunto de prácticas socioculturales que surgen a partir de la relación que se establece entre población local y visitantes. Antropológicamente las festividades han sido analizadas como un espacio para que las comunidades se vean, se piensen, momentos para la reflexividad, cancelar el Día del Tlachiquero o modificarlo implicaría perder dicha oportunidad, por ello a pesar de los inconvenientes, la población de Tepetlaoxtoc no quiere que dejen de acudir a tal festividad los jóvenes, porque se siguen viendo, pensando o reflexionando en la conducta de los visitantes.

Si bien la historia contada en el performance-ritual del Día del Tlachiquero aludía o reflejaba la cosmovisión del pueblo que representaba su tradición y memoria históricas, o bien una identidad colectiva que se recreaba sin cesar, la alteración ocasionada por la llegada de los jóvenes que buscan la oportunidad de realizar excesos, no causa mella en su eficacia simbólica porque sigue revelándose la cosmovisión, tradición e identidad de la colectividad visitada. Antes y después se trata de actos y juegos de espejos de dramatización; de una exhibición de cómo nos representamos a nosotros mismos, cómo deseamos ser y cómo queremos que los demás nos definan. De este modo los contenidos culturales tradicionales que el Día del Tlachiquero dramatizaba no tienen una reificada existencia a priori, no están fijados de una vez 
por todas, son creados, negociados, influidos y entremezclados por diversas ideologías. Con base en Weber particularmente la acción humana de estar a favor de la llegada de los visitantes evidencia nexos que pueden explicarse por la comprensión, de un modo intersubjetivo y, por lo tanto, plenamente objetivo.

Desde una posición dialógica se observa la negociación al interior de Tepetlaoxtoc entre quienes proponen que la fiesta se realice en un recinto ferial fuera de la localidad y en otra fecha, para que ahí se les venda pulque y se les ofrezcan espectáculos a los turistas y quiénes se niegan aduciendo que se rompería un ciclo mágico-religioso que pondría en peligro la seguridad de los habitantes de este municipio.

Institucionalizar el Día del Tlachiquero para convertirlo oficialmente en la Feria del Pulque puede ser una posibilidad para evitar sus impactos y con ello la molestia de la población local, pero es algo que se sigue negociando ya que robustece los valores compartidos y la moral sexual local. Como respuesta, localmente se implementan algunas medidas para mitigar los impactos más visibles, sin oponerse a la llegada de visitantes para seguir contando con el espacio social en el que el modelo de masculinidad se refuerza.

A partir de la perspectiva antropológica de la cultura, los elementos clave son la heterogeneidad y complejidad de las comunidades y la necesidad de tener en cuenta las culturas y perspectivas de las subcomunidades que pueden estar basadas, por ejemplo, en grupos étnicos, de género, profesionales o de interés. Dentro de cualquier comunidad, habrá una variedad de perspectivas hacia el turismo y estas perspectivas emanarán de los valores de las personas y las normas sociales, entre otras influencias. Desde una mirada antropológica, hay mucho en común entre el estudio de la cultura y el estudio de comunidades que están compuestas de seres humanos complejos. La comprensión de los patrones de comportamiento de los residentes y de los turistas puede proporcionar una idea de por qué ciertos impactos son más importantes para los residentes que otros en la comunidad o cuando menos tolerables en virtud de la obtención de beneficios simbólicos.

Finalmente, debido a que se trata de un estudio cualitativo se hizo uso de procedimientos que hacen menos comparables las observaciones en el tiempo y en diferentes circunstancias culturales, es decir, este método busca menos la generalización de los resultados, entendido como el grado en el cual los hallazgos de un estudio son aplicables a otras muestras o poblaciones, requiere interpretarse desde otra perspectiva que valore la naturaleza de la investigación cualitativa, cuyos resultados no buscan obtener leyes o principios, sino, examinar comprensivamente a los actores y contextos involucrados en el tema de estudio. Así la transferibilidad de los resultados dependerá de calidad metodológica en su ejecución.

\section{REFERENCIAS BIBLIOGRÁFICAS}

Akis, S., Peristianis, N., \& Warner, J. (1996). Residents' attitudes to tourism development: the case of Cyprus. Tourism management, 17(7), 481-494.

Andereck, K. L., \& Vogt, C. A. (2000). The relationship between residents' attitudes toward tourism and tourism development options. Journal of Travel research, 39(1), 27-36.

Andereck, K.L., Valentine, K., Knopf, R., \& Vogt, C. (2005). Residents' perceptions of community tourism impacts. Annals of Tourism Research, 32(4), 1056-1076. 
Ap, J., \& Crompton J. L. (1993). Residents' Strategies for Responding to Tourism Impacts. Journal of Travel Research, 33(1), 47-50.

Ap, J., \& Crompton, J. L. (1998). Developing and testing a tourism impact scale. Journal of Travel Research, 37 (2), 120-130.

Besculides, A., Lee, M. E., \& McCormick, P. J. (2002). Residents' perceptions of the cultural benefits of tourism. Annals of tourism research, 29(2), 303-319.

Blau, P. (1964). Exchange and power in social life. New York, NY: Wiley.

Butler, R. (1980). The concept of the tourist area cycle of evolution: implications for the management of resources. Canadian Geographer, 24(1), 5-12.

Choi, C., \& Murray, I. (2010). Resident attitudes towards sustainable community tourism. Journal of Sustainable Tourism, 18(4), 575-594.

Choi, H., \& Sirakaya, E. (2005). Measuring residents' attitude toward sustainable tourism: development of sustainable tourism attitude scale. Journal of Travel Research, 43(4), 380-394.

CONEVAL, (2015). Informe Anual Sobre La Situación de Pobreza y Rezago Social. Recuperado de http://www.sedesol.gob.mx/work/models/SEDESOL/Informes pobreza/2014/ Municipios/Mexico/Mexico 093.pdf

Deery, M., Jago, L., \& Fredline, L. (2012). Rethinking social impacts of tourism research: A new research agenda. Tourism Management, 33(1), 64-73.

Doxey, G. V. (1975). A Causation Theory of Visitor-Resident Irritants: Methodology and Research Inferences, in Proceedings of the $6^{\text {th }}$, Annual Conference of the Travel Research Association, Travel Research Association ed., Travel Research Association, San Diego, CA., 195-198

Dyer, P., Gursoy, D., Sharma, B., \& Cater, J. (2007). Structural modeling of resident perceptions of tourism and associated development on the Sunshine Coast, Australia. Tourism Management, 28(2), 409-422.

Emerson, R. (1976). Social exchange theory. Annals of Sociology, 2, 335-362.

Escriche, E. (2011) Lloret sale a la calle contra el turismo de borrachera. Opinión. Público. Recuperado de http://www.publico.es/espana/lloret-sale-calle-turismo-borrachera. html.

Faulkner, B., \& Tideswell, C. (1997). A framework for monitoring community impacts of tourism. Journal of Sustainable Tourism, 5(1), 3-28.

Fishbein, M., \& Ajzen, I. (1975). Belief, attitude, intention and behavior: An introduction to theory and research. Reading, MA: Addison-Wesley.

Fredline, E., \& Faulkner, B. (2000). Host community reactions: a cluster analysis. Annals of Tourism Research, 27(3), 763-784.

Geertz, C. (1987). La interpretación de las culturas. Barcelona: Gedisa.

Guber, R. (2004). El salvaje metropolitano: reconstrucción del conocimiento social en el trabajo de campo. Buenos Aires: Paidós.

Gunn, C.A. (1988). Vacationscape: Designing Tourist Regions. Nueva York, NY: Van Nostrand Reinhold.

Gursoy, D. Jurowski, C. y Uysal, M. (2002). Resident attitudes: A Structural Modeling Approach Residents. Annals of Tourism Research, 29(1), 79-105. 
Gursoy, D., \& Kendall, K. W. (2006). Hosting mega events: Modeling locals' support. Annals of tourism research, 33(3), 603-623.

Gursoy, D., \& Rutherford, D. G. (2004). Host attitudes toward tourism: An improved structural model. Annals of tourism Research, 31(3), 495-516.

Gursoy, D., Jurowski, C., \& Uysal, M. (2002). Resident attitudes: A structural modeling approach. Annals of tourism research, 29(1), 79-105.

Haralambopoulos, N., \& Pizam, A. (1996). Perceived impacts of tourism: The case of Samos. Annals of tourism Research, 23(3), 503-526.

Hernández, S. A., Cohen, J., \& García, H. L. (1996). Residents' attitudes towards an instant resort enclave. Annals of tourism research, 23(4), 755-779.

Huh, C., \& Vogt, C. A. (2008). Changes in residents' attitudes toward tourism over time: a cohort analytical approach. Journal of Travel Research, 46(4), 446-455.

Hunt, C., \& Stronza, A. (2014). Stage-based tourism models and resident attitudes towards tourism in an emerging destination in the developing world. Journal of Sustainable Tourism, 22(2), 279-298.

Johnson, J. D., Snepenger, D. J., \& Akis, S. (1994). Residents' perceptions of tourism development. Annals of tourism research, 21(3), 629-642.

Jurowski, C., \& Gursoy, D. (2004). Distance effects on residents' attitudes toward tourism. Annals of Tourism Research, 31(2), 296-312.

Kayat, K. (2002). Power, social exchanges and tourism in Langkawi: rethinking resident perceptions. International Journal of Tourism Research, 4(3), 171-191.

Lankford, S. V. (1994). Attitudes and perceptions toward tourism and rural regional development. Journal of travel research, 32(3), 35-43.

Lankford, S. V., \& Howard, D. R. (1994). Developing a tourism impact attitude scale. Annals of tourism research, 21(1), 121-139.

Lankford, S. V., Williams, A. L., \& Knowles-Lankford, J. (1997). Perceptions of outdoor recreation opportunities and support for tourism development. Journal of Travel Research, 35(3), 65-69.

Liu, J. C., Sheldon, P. J., \& Var, T. (1987). Resident perception of the environmental impacts of tourism. Annals of Tourism Research, 14(1), 17-37.

Mathieson, A., \& Wall, G. (1982), Tourism: economic, physical and social impacts. Londres, Reino Unido: Longman.

Milman, A., \& Pizam, A. (1988). Social impacts of tourism on central Florida. Annals of tourism research, 15(2), 191-204.

Monterrubio, J.C., \& Andriotis, K. (2014). Social representations and community attitudes towards spring breakers. Tourism Geographies. International Journal of Tourism Space, Place and Environment, 16(2), 288-302.

Northcote, J., \& Macbeth, J. (2005). Limitations of Resident Perception Surveys for Understanding Tourism Social Impacts: The Need for Triangulation. Tourism Recreation Research, 30(2), 43-54.

Perdue, R. R., Long, P. T., \& Allen, L. (1990). Resident support for tourism development. Annals of tourism Research, 17(4), 586-599.

Pitt-Rivers, J., (1979). Antropología del honor o política de los sexos, ensayos de antropología mediterranea. Barcelona: Crítica. 
Rivera Pérez, R. (2013). Bandidos, arrieros y machos contemporáneos del Acolhuacán Septentrional. Múltiples espacios para la construcción de la masculinidad: (Tesis doctoral). Universidad Autónoma Metropolitana. México.

Ross, G. F. (1992). Resident perceptions of the impact of tourism on an Australian city. Journal of Travel Research, 30(3), 13-17.

Sierra, E. (7 de agosto de 2017). Turismofobia, un presunto delito de odio. La Vanguardia. Recuperado de http://www.lavanguardia.com/local/barcelona/ 20170807/43325406588/turismofobia-barcelona-colau-odio-xenofobia-arran-juegos-olimpicos-1992-turistas-bus-turistico-asalto.html.

Sharma, B., Dyer, P., Carter, J., \& Gursoy, D. (2008). Exploring residents' perceptions of the social impacts of tourism on the Sunshine Coast, Australia. International Journal of Hospitality and Tourism Administration, 9(3), 288-311.

Sharpley, R. (2014). Host perceptions of tourism: A Review of the research. Tourism Management, 42, 37-49.

Small, K. (2007). Social dimensions of community festivals: an application of factor analysis in the development of the social impact perception (SIP) scale. Event Management, 11(1), 45-55.

Smith, M., \& Krannich, R. (1998). Tourism dependence and resident attitudes. Annals of Tourism Research, 25(4), 783-802.

Turner, V. (2007). La selva de los símbolos. Ciudad de México: Siglo XXI.

Vargas-Sánchez, A., Porras-Bueno, N., \& de los Ángeles Plaza-Mejía, M. (2011). Explaining residents' attitudes to tourism: Is a universal model possible? Annals of tourism research, 38(2), 460-480.

Velasco, H., \& Díaz de Rada, A. (1997). La lógica de la investigación etnográfica. Un modelo de trabajo para etnógrafos de la escuela. Madrid: Trotta.

Wang, Y., \& Pfister, R. (2008). Residents' attitudes toward tourism and perceived personal benefits in a rural community. Journal of Travel Research, 47(1), 84-93.

Weaver, D. B., \& Lawton, L. J. (2001). Resident perceptions in the urban-rural fringe. Annals of tourism research, 28(2), 439-458.

Weaver, D. B., \& Lawton, L. J. (2013). Resident perceptions of a contentious tourism event. Tourism Management, 37, 165-175.

Weber, Max (1964). Economía y sociedad. Esbozo de sociología comprensiva. Ciudad de México: Fondo de Cultura Económica

Williams, J., \& Lawson, R. (2001). Community issues and resident opinions of tourism. Annals of Tourism Research, 28(2), 269-290.

Woosnam, K. M. (2012). Using emotional solidarity to explain residents' attitudes about tourism and tourism development. Journal of Travel Research, 51(3), 315-327.

Yen, I., \& Kerstetter, D. (2009). Tourism impacts, attitudes and behavioral intentions. Tourism Analysis, 13(56), 545-564.

Yu, C. P., Chancellor, H. C., \& Cole, S. T. (2011). Measuring residents' attitudes toward sustainable tourism: A reexamination of the sustainable tourism attitude scale. Journal of Travel Research, 50(1), 57-63.

Zamani-Farahani, H., \& Musa, G. (2012). The relationship between Islamic religiosity and residents' perceptions of socio-cultural impacts of tourism in Iran: Case studies of Sare'in and Masooleh. Tourism Management, 33(4), 802-814. 